\title{
Estrutura de um solo construído cultivado com diferentes espécies de poáceas
}

\author{
Structure of a constructed soil cultivated with different species of poaceae
}

\section{Lizete Stumpf $^{* *}$ Eloy Antonio Pauletto ${ }^{\mathrm{II}}$ Rafaela Costa de Castro ${ }^{\mathrm{I}}$ Luiz Fernando Spinelli Pinto ${ }^{\mathrm{II}}$ Flavia Fontana Fernandes ${ }^{\text {II }}$ Fabrício da Silva Barboza ${ }^{\mathrm{III}}$ Ândrea Machado Pereira Franco ${ }^{\mathrm{I}}$ Fernanda Coelho Gonçalves ${ }^{\mathrm{I}}$}

RESUMO

Na mineração de carvão a céu aberto, a superfície é completamente suprimida de vegetação e as camadas de solo e rochas que recobrem os bancos de carvão são removidas, causando drásticas alterações no perfil do solo original. Este trabalho teve como objetivo avaliar a influência de diferentes espécies de poáceas na recuperação da estrutura de um solo construído, como parte da estratégia de recuperação de áreas degradadas por mineração de carvão. Os tratamentos avaliados foram: T1 - Hemarthria altissima, T2 - Paspalum notatum, T3 Cynodon dactilon, T4 - Urochloa brizantha. Como testemunha, utilizou-se o solo construído sem cobertura vegetal, denominada T5. Foram coletadas amostras de solo não preservadas $e$ preservadas nas camadas de 0,00-0,10m e 0,10-0,20m, para as determinações da porcentagem de macroagregados $e$ microagregados, diâmetro médio ponderado, carbono orgânico, densidade e macroporosidade do solo construído. Os resultados obtidos foram analisados aos 05, 41 e 78 meses após o estabelecimento do experimento. Aos 78 meses de condução do experimento, observa-se ação positiva das quatro espécies vegetais na recuperação da agregação do solo construído, com destaque para a Urochloa brizantha que proporcionou a menor densidade do solo e um dos maiores valores de macroporosidade. As poáceas proporcionaram uma melhoria na estruturação do solo construído, principalmente a partir dos 41 meses de condução do experimento, quando houve um incremento nos valores de DMP, principalmente na camada de 0,00-0,10m. Os teores de carbono orgânico foram incrementados ao longo do tempo, entretanto, isso não se refletiu em aumento do DMP aos 78 meses.

Palavras-chave: Hemarthria altíssima, Paspalum notatum, Cynodon dactilon, Urochloa brizantha, recuperação de área degradada.

\begin{abstract}
In surface coal mining the vegetation is completely suppressed and layers of soil and rocks that cover the coal seams are removed, causing drastic changes in the original soil profile. This study aimed to evaluate the influence of different species of grasses in the recovery of a constructed soil structure as part of the strategy of reclamation for coal mining. The treatments were: T1 - Hemarthria altissima, T2 - Paspalum notatum, T3 Cynodon dactilon, T4 - Urochloa brizantha. As a control, a bare constructed soil was used, called T5. Disturbed and undisturbed soil samples were collected in 0.00-0.10m and 0.10-0.20m layers for the determination of the percentage of macroaggregates and microaggregates, mean weight diameter, organic carbon, bulk density and macroporosity. The results were evaluated at 05, 41 and 78 months after the establishment of the experiment. After 78 months of conducting the experiment, positive action of the four plant species in the reclamation of soil aggregation was observed, especially for Urochloa brizantha that showed the lowest bulk density and the highest values of macroporosity. The species provided a structural improvement in the constructed soil, mainly after 41 months of conducting the experiment when there was an increase in the values of $M W D$, mainly in the 0.00 to 0.1 m layer. The organic carbon contents were increased over time, however, did not result in increased of MWD at 78 months.
\end{abstract}

Key words: Hemarthria altíssima, Paspalum notatum, Cynodon dactilon, Urochloa brizantha, reclamation of degraded land.

\section{INTRODUÇÃO}

Na mineração de carvão a céu aberto, a superfície é completamente suprimida de vegetação e

IPrograma de Pós-graduação em Agronomia, Universidade Federal de Pelotas (UFPel), Rua Campus Universitário Capão do Leão s/n, 96900-010, Capão do Leão, RS, Brasil. E-mail: zete.stumpf@gmail.com. *Autor para correspondência.

"Departamento de Solos, UFPel, Capão do Leão, RS, Brasil.

IIIPrograma de Pós-graduação em Manejo e Conservação do Solo e da Água, UFPel, Capão do Leão, RS, Brasil.

$$
\text { Recebido 12.11.13 Aprovado 08.05.14 Devolvido pelo autor 10.07.14 }
$$


as camadas de solo e rochas que recobrem os bancos de carvão são removidas, causando drásticas alterações no perfil do solo original (USSIRI \& LAL, 2005). Esse processo provoca a mistura dos horizontes do solo e rompe os agregados, expondo suas superfícies organominerais à ação de decompositores (WICK \& DANIELS, 2009). Além disso, a camada de solo reposta frequentemente é compactada pelas máquinas utilizadas na recomposição topográfica da área minerada (LIPIEC et al., 2003), afetando negativamente o movimento da água, a aeração e o desenvolvimento radicular das plantas ao longo do novo perfil construído (SENCINDIVER \& AMMONS, 2000).

A revegetação de solos construídos é uma importante estratégia de mitigação dos impactos negativos da mineração (USSIRI \& LAL, 2005), pois as plantas modificam e melhoram a estrutura do solo, principalmente quando suas raízes crescem em camadas densas, deixando poros ou canais, denominados bioporos, após a sua morte e decomposição (YUNUSA \& NEWTON, 2003).

Diferentemente do cenário agrícola, o entendimento da dinâmica de agregação e acumulação de matéria orgânica em solos minerados ao longo do tempo ainda são pouco compreendidos pela comunidade científica (WICK et al., 2010), principalmente no Brasil, onde os trabalhos ainda são escassos. Nesse contexto, o presente trabalho tem como objetivo avaliar a influência de diferentes espécies de poáceas na recuperação da estrutura de um solo construído, como parte da estratégia de recuperação de áreas degradadas por mineração de carvão.

\section{MATERIAL E MÉTODOS}

O estudo foi realizado em uma área de mineração de carvão, pertencente à Companhia Riograndense de Mineração (CRM), localizada em Candiota/RS. A camada de solo reposta na área experimental é procedente de um horizonte $\mathrm{B}$ do solo natural da área pré-minerada, um Argissolo Vermelho Eutrófico típico, apresentando a seguinte caracterização: cor vermelho escura (2,5 YR 3,5/6), teor de matéria orgânica de $1,15 \%, \mathrm{pH}$ em água de 5,6, teores de cálcio, magnésio e alumínio de 2,65, 2,11 e $1,28 \mathrm{cmol} \mathrm{kg}^{-1}$, respectivamente, CTC de $8,69 \mathrm{mg} \mathrm{kg}^{-1}$ e classe textural argilosa $(315,209$, $476 \mathrm{~g} \mathrm{~kg}^{-1}$ de areia, silte e argila, respectivamente) na camada de 0,00-0,15m do solo construído.

O solo foi construído no início de 2003 e o experimento instalado em novembro/dezembro de 2003, apresentando parcelas de $20 \mathrm{~m}^{2}(5 \mathrm{mx} 4 \mathrm{~m})$, em delineamento de blocos ao acaso, com quatro repetições. Antes da instalação do experimento, em face da área se encontrar extremamente compactada, devido à grande circulação de máquinas durante a construção do solo (caminhões carregados com aproximadamente $20 \mathrm{Mg}$ de terra vegetal e tratores de esteira metálica do modelo D8T da Caterpillar ${ }^{\circledR}$ com peso de $38 \mathrm{Mg}$, potência de $259 \mathrm{KW}$, comprimento e largura da esteira sobre o solo de 3,20 e 0,56m, respectivamente, e área de contato das esteiras com o solo de $3,6 \mathrm{~m}^{2}$ ), o solo construído foi escarificado com patrola a uma profundidade aproximada de $0,15 \mathrm{~m}$, seguido por calagem correspondente a $10,4 \mathrm{Mg} \mathrm{ha}^{-1}$ de calcário com PRNT de $100 \%$ e uma adubação de $900 \mathrm{~kg} \mathrm{ha}^{-1}$ da fórmula 5-20-20, com base em resultados obtidos pela análise de solo.

Foram estudadas espécies vegetais perenes, incluindo gramíneas e leguminosas (Lotus pedunculatus cv 'Makú' e Arachis Pintoi), por estas apresentarem capacidade de se manterem na área ao longo do tempo, bem como pela alta capacidade de adição de biomassa ao solo, entretanto, as leguminosas não se estabeleceram na área. Assim, os tratamentos estudados foram: T1-Hemarthria altíssima; T2Paspalum notatum cv. 'Pensacola'; T3-Cynodon dactilon cv 'tifton' e T4 - Urochloa brizantha. Como testemunha, foi utilizado o solo construído sem cobertura vegetal, denominado T5, localizado em uma área adjacente à área experimental.

Aos 78 meses de condução do experimento (Junho de 2010), foram coletadas 80 amostras não preservadas nas camadas de $0,00-0,10 \mathrm{~m}$ e $0,10-0,20 \mathrm{~m}$ para a determinação da distribuição de agregados estáveis em água, em diferentes classes de tamanho, do diâmetro médio ponderado de agregados estáveis em água (DMP) e do teor de matéria orgânica do solo. Também foram coletadas 80 amostras preservadas em ambas as camadas para a determinação da densidade do solo (BLAKE \& HARTGE, 1986) e da macroporosidade (EMBRAPA, 2011).

As amostras foram passadas na peneira de malha $9,5 \mathrm{~mm}$, com base no peneiramento úmido, seguindo o método descrito por KEMPER \& ROSENAU (1986) e adaptado por PALMEIRA et al. (1999), utilizando o aparelho de oscilação vertical. Os intervalos das classes dos agregados foram: $\mathrm{C} 1$ : 9,524,76mm; C2: 4,76-2,0mm; C3: 2,00-1,00mm; C4: 1,00-0,25mm; C5: 0,25-0,105mm e C6: $<0,105 \mathrm{~mm}$. A partir dessas classes, os agregados foram separados em macroagregados, ou seja, agregados maiores que $0,25 \mathrm{~mm}$, e microagregados, agregados menores que $0,25 \mathrm{~mm}$, de acordo com TISDALL \& OADES (1982). O teor de carbono no solo foi determinado 
pelo método de combustão Walkley-Black, em amostras passadas na peneira de $2 \mathrm{~mm}$, segundo TEDESCO et al. (1995).

Os dados foram submetidos à análise de variância $(\mathrm{P}<0,05)$ e, havendo diferenças significativas, as médias dos tratamentos $\mathrm{T} 1$ a $\mathrm{T} 4$ foram comparadas pelo teste de Tukey $(\mathrm{P}<0,05)$. Considerando $o$ fato de a testemunha T5 não fazer parte do delineamento experimental, não se aplicou procedimentos estatísticos em relação aos demais tratamentos. Para avaliar a influência das diferentes poáceas na recuperação da agregação do solo construído ao longo do tempo, analisaram-se os resultados obtidos aos 05, 41 e 78 meses após o estabelecimento do experimento, aplicando a técnica de análise de medidas repetidas, por meio do procedimento proc mixed. Todas as análises foram realizadas por meio do software estatístico SAS (STATISTICAL ANALYSIS SYSTEM, 1985).

\section{RESULTADOS E DISCUSSÃO}

Após 78 meses de condução do experimento, os tratamentos com poáceas não mostraram diferenças significativas entre si na camada de $0,00-0,10 \mathrm{~m}$. Todavia, o potencial de recuperação da estrutura do solo por parte das espécies vegetais é evidenciado, quando se comparam os tratamentos (T1 a T4) com a testemunha (T5), isto é, observase que todos os tratamentos proporcionaram valores superiores de macroagregados (11,12 a 16,86\%) e inferiores de microagregados $(24,81$ a $37,60 \%)$ e um valor superior de DMP (6,63 a 33,13\%), em relação ao solo construído sem cobertura vegetal (Tabela 1).

$\mathrm{O}$ efeito positivo dos tratamentos na superfície do solo construído também é corroborado quando se observam os menores valores de densidade do solo $(2,70$ a $7,43 \%)$ e os maiores valores de macroporosidade $(18,18 \%)$, apresentados pela maioria

Tabela 1 - Valores médios das porcentagens de macroagregados e microagregados, diâmetro médio ponderado (DMP) de agregados estáveis em água, teor de carbono orgânico (CO), densidade do solo (Ds) e macroporosidade (Ma) em solo construído, cultivado com diferentes poáceas e suas diferenças em relação à testemunha (solo sem cobertura vegetal) nas camadas de 0,00-0,10 e 0,10$0,20 \mathrm{~m}$, aos 78 meses de condução do experimento.

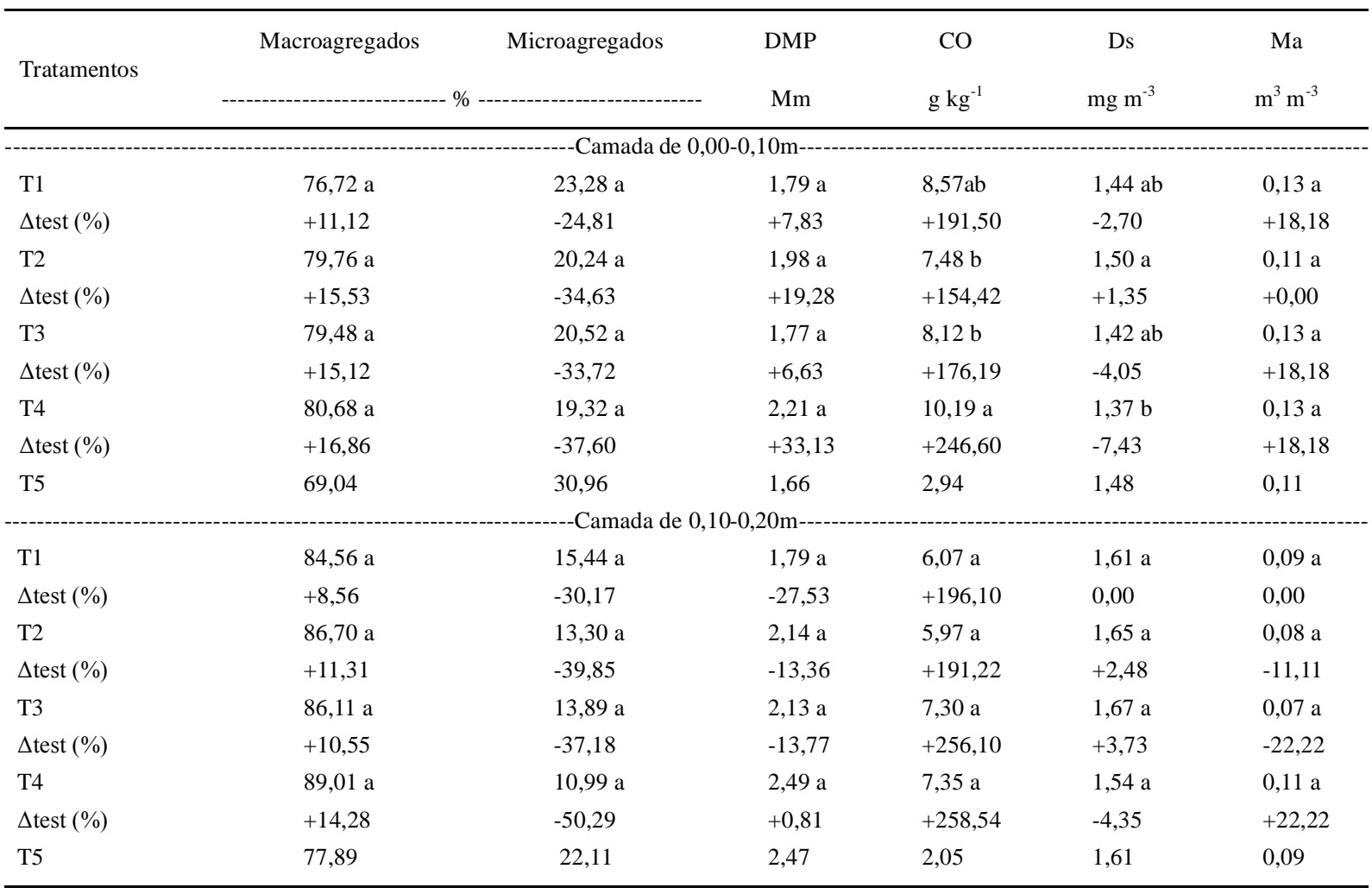

T1: Hemarthria altíssima; T2: Paspalum notatum; T3: Cynodon dactilon; T4: Urochloa brizantha; T5: solo construído sem cobertura vegetal. Letras minúsculas iguais na coluna, em cada camada, não diferem entre si para o teste de Tukey $(\mathrm{P}<0,05)$. $\Delta$ test $(\%)$ : aumento $(+)$ ou diminuição (-) em relação à testemunha T5.

Ciência Rural, v.44, n.12, dez, 2014. 
dos tratamentos em relação ao T5 (Tabela 1). Esse resultado pode estar relacionado ao desenvolvimento radicular das plantas avaliadas, pois, segundo LIMA et al. (2012), culturas com sistema radicular agressivo propiciam a formação de bioporos com ampla variação de tamanho, constituindo-se em uma alternativa para a minimização dos efeitos da compactação do solo.

$\mathrm{Na}$ tabela 1, observa-se ainda que o tratamento T4 proporcionou o maior teor de carbono orgânico (CO) em relação aos demais tratamentos na camada de 0,00-0,10m. Entretanto, este resultado não se refletiu em diferenças significativas dos valores de DMP entre os tratamentos, embora SIX et al. (2000) afirmem que o incremento de $\mathrm{CO}$ no solo influencia no aumento do DMP. Neste estudo, a correlação entre os valores de DMP e CO não foi significativa, indicando que o teor de $\mathrm{CO}$ ainda não é o fator preponderante na agregação do solo construído.

Assim como observado na camada de 0,00 0,10m do solo construído, os tratamentos com poáceas também não diferiram entre si na camada de 0,10 0,20m, aos 78 meses de condução do experimento. Observa-se também que, na camada subsuperficial, todos os tratamentos mostraram valores superiores de macroagregados (8,56 a 14,28\%) e inferiores de microagregados $(30,17$ a $50,29 \%)$ em relação à testemunha (T5). Entretanto, ao se avaliar os valores de DMP, apenas o tratamento T4 proporcionou um incremento em relação ao T5 $(0,81 \%)$ (Tabela 1$)$. Este resultado possivelmente deve-se a um menor desenvolvimento radicular das demais espécies vegetais nesta camada do solo construído, por conta da maior compactação que esta apresenta, observada através dos valores de densidade do solo muito superiores a $1,40 \mathrm{Mg} \mathrm{m}^{-3}$ e os valores de macroporosidade abaixo dos $0,10 \mathrm{~m}^{3} \mathrm{~m}^{-3}$ (Tabela 1), considerados críticos ao adequado desenvolvimento de plantas (REICHERT et al., 2007; TORMENA et al.,1998).

A maior compactação da camada de 0,10$0,20 \mathrm{~m}$ em relação à camada de $0,00-0,10 \mathrm{~m}$ do solo construído ainda é um reflexo do tráfego intenso de máquinas durante a recomposição topográfica da área minerada. Estruturas massivas ocorrem em solos argilosos compactados, quando partículas de argila são comprimidas em grandes blocos coesos (TOPP et al., 1997), em face do tráfego de máquinas em condições de umidade inadequada.

Contudo, a potencialidade das poáceas na recuperação da agregação de um solo degradado pela mineração é observada quando se avaliam os valores da porcentagem de macroagregados, microagregados e DMP em diferentes períodos de condução do experimento (aos 05, 41 e 78 meses) (Tabela 2).
Ressalta-se que a maior porcentagem de macroagregados (T2 a T4) e os maiores valores de DMP (T1 a T4) observados na camada de 0,00-0,10m, aos 05 meses de condução do experimento (Tabela 2), não se deve à ação biológica das plantas de cobertura, mas pelo efeito da compactação inicial, ocasionada durante a construção do solo. De acordo com HORN et al. (1995), a compactação do solo, causada pelo tráfego de máquinas pesadas, geralmente resulta em deterioração da estrutura do solo, expressa pela formação de agregados grandes, porém pouco porosos. Agregados formados por compressão e não por ação biológica já foram relatados por CARPENEDO \& MIELNICZUK (1990) e BERGAMIN et al. (2010) em solos argilosos. Todavia, dos 05 aos 41 meses de condução do experimento, observa-se a diminuição significativa da porcentagem de macroagregados (T2 a T4) e dos valores de DMP (T1 a T4), e consequentemente um incremento significativo dos valores de microagregados (T2 a T4) (Tabela 2). Possivelmente, este resultado seja um reflexo do início da atividade biológica das plantas de cobertura, associado aos ciclos de umedecimento e secagem, comuns em solos argilosos. Estes dois fatores, segundo HORN (1990), podem alterar agregados formados naturalmente e/ou antropogênicamente.

$\mathrm{O}$ incremento significativo nos valores de DMP observado dos 41 aos 78 meses na camada de $0,00-0,10 \mathrm{~m}$ pode ser um indicador da atuação mais efetiva das poáceas na agregação do solo construído, com destaque para o T4 $(62,50 \%)$ e T2 $(45,59 \%)$ (Tabela 2). A agregação pode ser correlacionada com a massa e morfologia de raízes (BRONICK \& LAL, 2005), tanto pela liberação de exsudados orgânicos como pela compressão das partículas e remoção de água durante seu crescimento no espaço poroso do solo (PERIN et al., 2002).

Cabe ressaltar que o incremento significativo do teor de $\mathrm{CO}$ ao longo do tempo (principalmente dos 05 aos 41 meses) foi acompanhado pelo aumento dos valores de microagregados da maioria dos tratamentos, na camada superficial do solo construído (Tabela 2). Segundo TISDALL \& OADES (1982), o sistema radicular e as hifas de fungos podem atuar como núcleos de formação de microagregados e, por serem ligantes temporários, formam fragmentos recobertos por mucilagens, dando origem a novos agregados.

$\mathrm{O}$ menor valor de $\mathrm{CO}$ verificado aos 05 meses de condução do experimento, em relação aos demais períodos (Tabela 2), deve-se à degradação ocasionada pela remoção dos horizontes do solo original durante a mineração e a utilização do horizonte B na construção do solo. De acordo com 
Tabela 2 - Porcentagem de macroagregados e microagregados, diâmetro médio ponderado (DMP) e teor de carbono orgânico (CO) de um solo construído, cultivado com diferentes poáceas nas camadas de $0,00-0,10 \mathrm{~m}$ a $0,10-0,20 \mathrm{~m}$, avaliada aos 05,41 e 78 meses de condução do experimento.

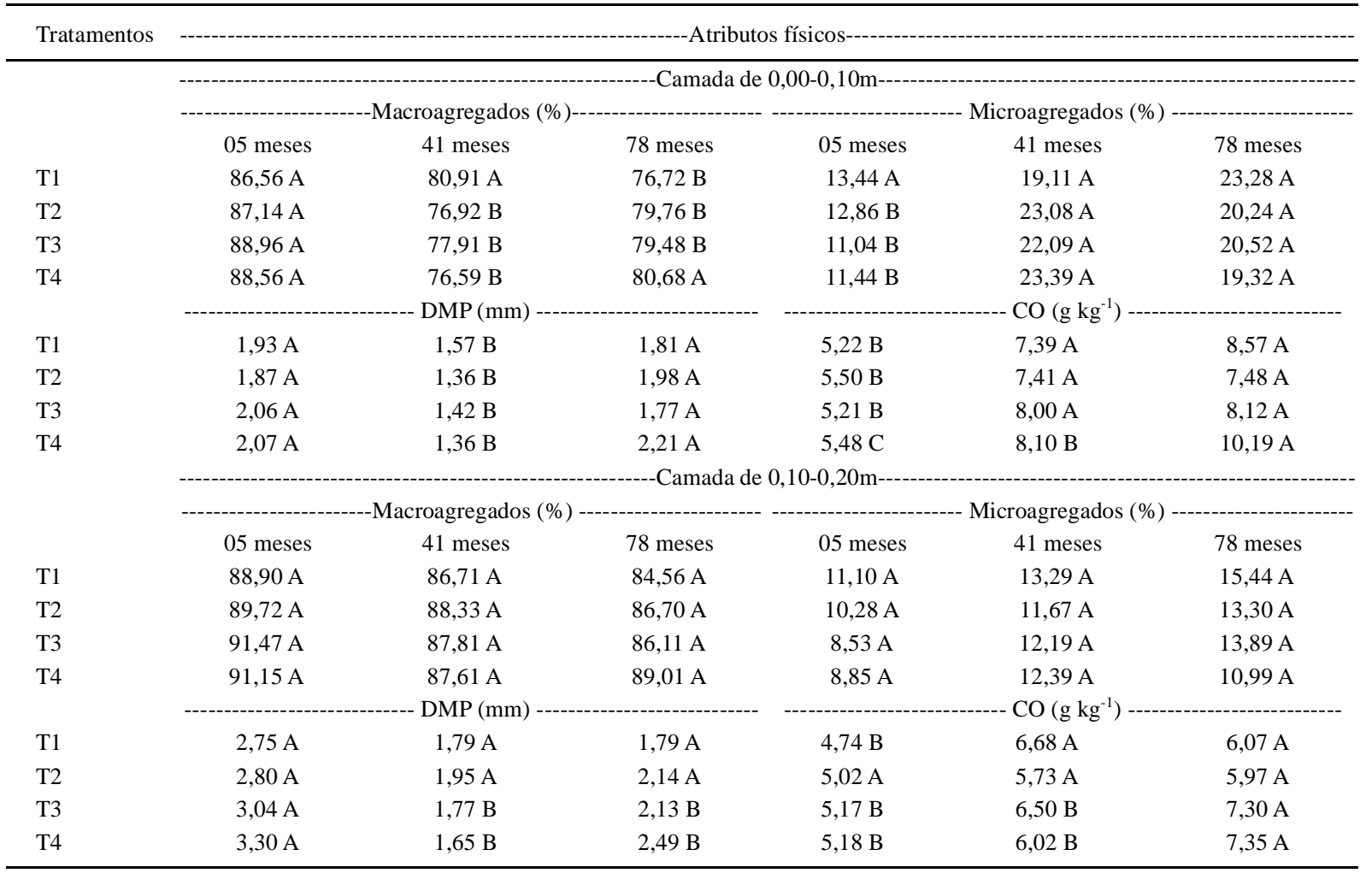

T1: Hemarthria altíssima; T2: Paspalum notatum; T3: Cynodon dactilon; T4: Urochloa brizantha. Letras maiúsculas iguais na linha, em cada camada, não diferem entre si para o teste de Tukey $(\mathrm{P}<0,05)$.

USSIRI \& LAL (2005), perdas de carbono podem ser atribuídas à mistura mecânica dos horizontes $\mathrm{A}$, $\mathrm{B}$, e $\mathrm{C}$ durante a remoção e manipulação do solo, bem como pela decomposição acelerada da matéria orgânica enquanto o solo fica exposto.

$\mathrm{Na}$ camada de $0,10-0,20 \mathrm{~m}$ do solo construído, observa-se que os tratamentos não indicaram diferenças significativas entre si para a porcentagem de macro e microagregados dos 05 aos 78 meses de avaliação (Tabela 2). Ao se avaliar os valores de DMP, observa-se que os tratamentos T3 e T4 diminuíram significativamente seus valores dos 05 aos 78 meses de condução do experimento (Tabela 2). Essas diferenças devem-se possivelmente aos efeitos dos ciclos de umedecimento e secagem, já que as condições físicas desta camada (elevada densidade e baixa macroporosidade) (Tabela 1) não permitem que o sistema radicular se desenvolva de forma adequada. De acordo com SEGUEL \& HORN (2006), os processos de umedecimento e secagem resultam em fendas orientadas verticalmente, que induzem a formação da estrutura em blocos. Por outro lado, estas rachaduras naturais futuramente podem fornecer caminhos para o crescimento radicular nas camadas mais duras do solo (BALL et al., 2005).

Os resultados deste estudo mostram que a recuperação dos atributos físicos do solo em áreas impactadas pela mineração do carvão a céu aberto ocorre de forma lenta, principalmente na camada subsuperficial do solo, que apresenta maior compactação. No entanto, espera-se que, no decorrer dos anos, o contínuo aporte de matéria orgânica das espécies vegetais, via aérea e subterrânea, atue de forma mais eficiente na formação de novos agregados, auxiliando na recuperação das condições edáficas do solo construído, principalmente em profundidade. Segundo VEZZANI \& MIELNICZUK (2011), o uso de gramíneas perenes pode recuperar a qualidade estrutural do solo, devido à permanência intacta dos restos de raízes das culturas e à ação da meso e macro fauna na fragmentação desses resíduos. 
Vale ressaltar que, no caso dos solos construídos, a recuperação é mais lenta do que em áreas agrícolas, pois a extração do carvão mineral promove a destruição total do perfil do solo, que levou milhares de anos para ser formada, diferente do solo agrícola que normalmente apresenta apenas a camada superficial $(0,00-0,20 \mathrm{~m})$ de solo desestruturado, se adotado um manejo inadequado.

\section{CONCLUSÃO}

Após 78 meses de condução do experimento, observa-se ação positiva das quatro espécies vegetais utilizadas na recuperação da agregação do solo construído, com destaque para a Urochloa brizantha, que proporcionou a menor densidade do solo e um dos maiores valores de macroporosidade.

Ao longo do tempo, as poáceas proporcionaram uma melhoria na estruturação do solo construído, principalmente a partir dos 41 meses de condução do experimento, quando houve um incremento nos valores de DMP, principalmente na camada de 0,00-0,10m.

Os teores de carbono orgânico foram incrementados ao longo do tempo, entretanto, isso não se refletiu em aumento do diâmetro médio ponderado de agregados aos 78 meses.

\section{AGRADECIMENTOS}

A Companhia Riograndense de Mineração (CRM), a Rede do Carvão, a Coordenação de Aperfeiçoamento de Pessoal de Nível Superior (CAPES) e ao Conselho Nacional de Desenvolvimento Científico e Tecnológico $(\mathrm{CNPq})$, pelo apoio e financiamento deste estudo.

\section{REFERÊNCIAS}

BALL, B.C. et al. The role of crop rotations in determining soil structure and crop growth conditions. Canadian Journal of Soil Science, v.85, n.5, p.557-577, nov. 2005. Disp.onível em: <pubs. aic.ca/doi/pdf/10.4141/S04-078>. Acesso em: 25 mar. 2013. doi: 10.4141/S04-078.

BRONICK, C.J.; LAL, R. Soil structure and management: a review. Geoderma, v.124, n.1, p.3-22, jan. 2005. Disponível em: <htpp:// www.sciencedirect.com/science/article /pii/S0016706104000898>. Acesso em: 10 abr. 2013. doi: 10.1016/j.geoderma.2004.03.005.

BERGAMIN, A.C. et al. Compactação em um Latossolo Vermelho distroférrico e suas relações com o crescimento radicular do milho. Revista Brasileira de Ciência do Solo, v.34, n.3, p.681-691, mai/jun. 2010. Disponível em:<http://www.scielo. $\mathrm{br} / \mathrm{scielo}$.php? pid=S0100-06832010000300009\& script $=\mathrm{sci}$ arttext>. Acesso em: 10 abr. 2013. doi: 10.1590/S010006832010000300009

BLAKE, G.R.; HARTGE, K.H. Bulk density. In: KLUTE, A. Methods of soil analysis: physical and mineralogical methods. $2^{\mathrm{N}}$.ed. Madison: American Society of Agronomy. 1986. p. $363-375$

CARPENEDO, V.; MIELNICZUCK, J. Estado de agregação e qualidade de agregados de Latossolos Roxos submetidos a diferentes sistemas de manejo. Revista Brasileira de Ciência do Solo, v.14, n.1, p.99-105, jan. 1990. CD.

EMBRAPA,Centro Nacional de Pesquisa de Solos. Manual de métodos de análise de solo. Rio de Janeiro: EMBRAPA CNPS, 2011. 230p.

HORN, R. Aggregate characterization as compared to bulk soil properties. Soil and Tillage Research, v.17, n.3, p.265-289, sept. 1990. Disponível em: <http://www.sciencedirect.com /science/ article/pii/016719879090041B >. Acesso em: 16 abr. 2013. doi: 10.1016/0167-1987(90)90041-B

HORN, R. et al. Soil compaction processes and their effects on the structure of arable soils and the environment. Soil and Tillage Research, v.35, n.1, p.23-36, aug. 1995. Disponível em: <http:// www.sciencedirect.com/science/article/pii/016719879500479C>. Acesso em 16 abr. 2013. doi: 10.1016/0167-1987(95)00479-C.

KEMPER, W.D.; ROSENAU, R.C. Aggregate stability and size distribution. In: KLUTE, A. Methods of soil analysis. 2.ed. Madison: American Society of Agronomy Soil Science Society of America, 1986. p.425-441.

LIMA, V.M.P. et al. Intervalo hídrico ótimo como indicador de melhoria da qualidade estrutural de Latossolo degradado. Revista Brasileira de Ciência do Solo, v.36, n.1, p.71-78, 2012. Disponível em: <http://www.scielo.br/scielo.php?pid=S010006832012000100008\&script=sci_arttext $>$. Acesso em: 06 jan. 2014. doi: 10.1590/S0100-06832012000100008.

LIPIEC, J. et al. Review of modelling crop growth, movement of water and chemicals in relation to topsoil and subsoil compaction. Soil and Tillage Research, v.73, n.1, p.15-29. oct. 2003. Disponível em: <http://www.sciencedirect.com /science/ article/pii/S0167198703000965>. Acesso em: 20 mar. 2013. doi: 10.1016/S0167-1987(03)00096-5.

PALMEIRA, P.R.T. et al. Agregação de um Planossolo submetido a diferentes sistemas de cultivo. Revista Brasileira de Ciência do Solo, v.23, n.2, p.189-195, 1999. CD.

PERIN, A. et al. Efeito da cobertura viva com leguminosas herbáceas perenes na agregação de um Argissolo. Revista Brasileira de Ciência do Solo, v.26, n.3, p.713-720, 2002. Disponível em: <http://sbcs.solos.ufv.br/solos/revistas/v26n3a16. pdf>. Acesso em: 20 mar. 2013.

REICHERT, J.M. et al. Compactação do solo em sistemas agropecuários e florestais: identificação, efeitos, limites críticos e mitigação. Tópicos em ciência do solo. Viçosa: Sociedade Brasileira de Ciência do Solo, 2007. p.49-134.

SEGUEL, O.; HORN, R. Structure properties and pore dynamics in aggregate beds due to wetting-drying cycles. Journal of Plant Nutrition and Soil Science, v.169, n.2, p.221-232, Mar. 2006. Disponível em: <http://onlinelibrary.wiley.com/doi/10.1002/ jpln.200521854/pdf>. Acesso em: 16 maio, 2013. doi: 10.1002/ jpln.200521854.

SAS (STATISTICAL ANALYSIS SYSTEM). User's guide. 5.ed. Cary, N.C. 1985.

Ciência Rural, v.44, n.12, dez, 2014. 
SENCINDIVER, J.C.; AMMONS, J.T. Minesoil genesis and classification. In: BARNHISEL, R.I. et al. Reclamation of drastically disturbed lands. Madison, WI: American Society Agronomy, 2000. Ch.23. (Agronomy Series n.41.) p. 595-613.

SIX, J. et al. Soil structure and organic matter: I. Distribution of aggregate-size classes and aggregate-associated carbon. Soil Science Society of American Journal, v.64, n.2, p.681-689, mar. 2000. Disponível em: <https://dl.sciencesocieties.org/ publications/sssaj/abstracts /64/2/681>. Acesso em: 16 maio, 2013. doi: 10.2136/sssaj2000.642681x.

TEDESCO, M.J. et al. Análises de solo plantas e outros materiais. 2.ed. Rev. e ampl. Porto Alegre-RS: Departamento de Solos Universidade Federal do Rio Grande do Sul, UFRGS, 1995. 174p.

TISDALL, J.M.; OADES, J.M. Organic matter and water-stable aggregates in soils. European Journal of Soil Science, v. 33, p 141-163. Jun, 1982.

TOPP, G.C. et al. Physical attributes of soil quality. In GREGORICH, E.G.; CARTER, M.R. Soil quality for crop production and ecosystem health. New York, NY: Elsevier, 1997. p.21-58. (Developments in Soil Science, n.25).

TORMENA, C.A. et al. Caracterização do intervalo hídrico ótimo de um Latossolo Roxo sob plantio direto. Revista Brasileira de Ciência do Solo, v.22, n.1, p.573-581, 1998. Disponível em: <http://www.alice.cnptia.embrapa.br/bitstream/doc/335757/1/ v22n4a022.pdf>. Acesso em: 10 fev. 2013.

USSIRI, D.A.N.; LAL, R. Carbon sequestration in reclaimed minesoils. Critical Reviews in Plant Sciences, v.24, n.3, p.151165. Jul. 2005. Disponível em: <http://scholar.google.com.br/ sc holar?cluster $=10227267651636152080 \& \mathrm{hl}=\mathrm{ptPT} \&$ as_sdt=0,5\#> . Acesso em:16 maio, 2013. doi:10.1080/07352680591002147.

VEZZANI, F.M.; MIELNICZUK, J. Agregação e estoque de carbono em Argissolo submetido a diferentes práticas de manejo agrícola. Revista Brasileira de Ciência do Solo, v.35, n.1, p.213223, jan/fev. 2011. Disponível em: <http://www.redalyc.org/ articulo.oa?id=180219142020 >. Acesso em: 20 mar. 2013. doi: 180219142020

WICK, A.F.; DANIELS, W.L. Physical protection of organic matter in reclaimed coal mine soils of SW Virginia. In: BILLINGS LAND RECLAMATION SYMPOSIUM, 2009, Billings, MT. Published by ASMR, 3134 Montevesta Rd., Lexington, KY, 40502. Online. Disponível em: <http://landrehab.org/UserFiles/ DataItems/694B3577446231477067773D/Wick\%20et\%20 al.,\%202009\%20ASMR\%20Physical\%20protection>. Acesso em: 20 mar. 2013.

WICK, A.F. et al. Soil aggregation, organic matter and microbial dynamics under different amendments after 27 years of mine soil development. In: BARNHISEL, R.I.(Ed.). Proceeding of National Meeting of the American Society of Mining and Reclamation, Bridging Reclamation, Science and the Community. American Society for Mining and Reclamation, Pittsburgh, PA. 2010. p.13641386. Online. Disponível em: <http://www.asmr.us/Publications/ Conference\%20Proceedings/2010/papers/1364-Wick-VA>. Acesso em: 20 mar. 2013.

YUNUSA, I.A.M.; NEWTON, P.J. Plants for amelioration of subsoil constraints and hydrological control: the primerplant concept. Plant and Soil, v.257, n.2, p.261-281, mês. 2003. Disponível em: <http://link.springer.com/article/10.1023/ A:1027381329549\#page-1>. Acesso em: 16 abr. 2013. doi: $10.1023 / \mathrm{A} \cdot 1027381329549$ 